University of New Mexico

UNM Digital Repository

$1-1-2006$

\title{
Maternal condition and facultative sex ratios in populations with overlapping generations.
}

L.E. Schwanz

J.G. Bragg

Eric Charnov

Follow this and additional works at: https://digitalrepository.unm.edu/biol_fsp

Part of the Biology Commons

\section{Recommended Citation}

American Naturalist 168(4):521-530

This Article is brought to you for free and open access by the Academic Department Resources at UNM Digital Repository. It has been accepted for inclusion in Biology Faculty \& Staff Publications by an authorized administrator of UNM Digital Repository. For more information, please contact disc@unm.edu. 


\title{
Maternal Condition and Facultative Sex Ratios in Populations with Overlapping Generations
}

\author{
Lisa E. Schwanz, ${ }^{\star}$ Jason G. Bragg, ${ }^{\dagger}$ and Eric L. Charnov ${ }^{\ddagger}$
}

Department of Biology, University of New Mexico, Albuquerque, New Mexico 87131

Submitted December 5, 2005; Accepted June 21, 2006;

Electronically published August 31, 2006

Online enhancement: appendix.

\begin{abstract}
AвSTRACT: Facultative investment in offspring sex is related to maternal condition in many organisms. In mammals, empirical support for condition-dependent sex allocation is equivocal, and there is some doubt as to theoretical expectations. Much theory has been developed to make predictions for condition-dependent sex ratios in populations with discrete generations. However, the extension of these predictions to populations with overlapping generations (OLGs; e.g., mammals) has been limited, leaving doubt as to the specific prediction for maternal-condition-dependent sex ratios in mammals. We develop a population genetics model that incorporates maternal effects on multiple offspring fitness components in a population with OLGs. Using a rare-gene and evolutionarily stable strategy approach, we demonstrate that sex ratio predictions of this model are identical to those for equivalent discrete generations models. We show that the predicted sex ratios depend on the sex-specific ratio of $R_{\mathrm{o}}$ (offspring lifetime fitness) for offspring of good and poor mothers. This offspring lifetime fitness rule indicates that empirical research on conditional sex ratios should consider all three components of offspring $R_{\mathrm{o}}$ (juvenile survival, adult life span, and fertility).
\end{abstract}

Keywords: Trivers-Willard model, Charnov-Bull model, mammal sex ratios, overlapping generations, population genetics model.

Trivers and Willard (1973) first proposed that a mother may allocate to offspring of different sexes according to her condition. They suggested that in a polygynous mating system, reproductive success is highly skewed in males compared with females-only the few best males secure

\footnotetext{
* Corresponding author; e-mail: schwanz@unm.edu.

E-mail: jbragg@unm.edu.

‡E-mail: rlc@unm.edu.
}

Am. Nat. 2006. Vol. 168, pp. 521-530. (c) 2006 by The University of Chicago. 0003-0147/2006/16804-41478\$15.00. All rights reserved. reproductive opportunities. In such a system, a mother that is going to bear reproductively competitive offspring should have sons, assuming that she can alter sex ratio without compromising other fitness components (e.g., litter size). The model assumes that maternal condition affects offspring condition, that offspring condition is maintained until adulthood, and that offspring condition has a greater effect on a son's reproductive success than on that of a daughter. Reproductive competitive ability is the measure of offspring fitness in the Trivers-Willard (T-W) hypothesis.

Stimulated by the T-W ideas, Charnov and Bull (1977) extended the principle (condition-dependent male/female fitness effects should select for facultative sex ratios) to a wide variety of contexts (overviews in Charnov 1982, 1993). It was also soon realized that Ghiselin's (1969) sizeadvantage hypothesis for sex reversal made very similar male/female fitness assumptions. There is now a large literature on condition-dependent sex ratios in diverse organisms (see, e.g., de Jong and Klinkhamer 2005 for plants).

Although the T-W hypothesis was developed with ungulate mammals in mind, subsequent application of the model to mammals has led to inconsistent results (e.g., Hewison and Gaillard 1999; Cockburn et al. 2002; Sheldon and West 2004). While compelling examples of conditiondependent biases in offspring sex exist in mammals (e.g., Clutton-Brock et al. 1986; Cameron et al. 1999; Fisher 1999), support for the T-W hypothesis is often unclear in specific studies or when considering the results of such studies in aggregate. In some species, sex ratio biases are related to maternal condition, but the assumptions of the T-W hypothesis have not been tested (Hewison and Gaillard 1999). In other species, the assumptions of the model appear to be met, but no conditional sex ratio biases are observed (Krackow 1997; Bercovitch et al. 2000; Blanchard et al. 2005). Recent reviews of sex ratios in mammals (and birds) have substantially advanced our understanding of the diverse empirical results by highlighting the potential constraints on sex ratio adjustment, the interaction of multiple selective agents, and the proper measures of maternal 
condition (Cockburn et al. 2002; West and Sheldon 2002; Cameron 2004; Sheldon and West 2004; West et al. 2005). Many inconsistencies in studies of the T-W hypothesis may be attributed to a mismatch between theory and empiricism. That is, potentially, predictions are not met because of insufficient knowledge of the biology of the organisms (e.g., offspring fitness components) and how it relates to theoretical foundations. Alternatively, it may be because models do not explicitly take into account the complicated life histories of long-lived animals with overlapping generations (OLGs; Frank 1990; Pen and Weissing 2002).

Previous theoretical work on condition-dependent sex ratios has applied game theory (evolutionarily stable strategies) to population genetic models to demonstrate that (i) individuals in good condition should allocate more to the sex whose fitness increases more steeply with condition and (ii) optimal sex allocation depends on the frequency distribution of conditions in the population (Charnov et al. 1978, 1981; Charnov 1979; Bull 1981; Leimar 1996). Additionally, previous models have had great predictive success in a number of empirical studies of nonmammalian taxa (e.g., Charnov et al. 1978, 1981). The application of these models to mammals, however, has been complicated by the fact that most condition-dependent sex ratio models do not include OLGs or many other demographic features of mammals (Frank 1990; for OLG models, see Charnov and Dawson 1989; Leimar 1996; Pen et al. 1999; Pen and Weissing 2002).

There are several reasons why studying sex allocation in an OLG framework can provide insight into conditiondependent sex ratios in mammals. First, it has been shown that sex ratio predictions can depend on OLGs, for example, in seasonal populations and in response to population perturbation (Werren and Charnov 1978; West and Godfray 1997). Second, with OLGs, offspring compete for reproductive opportunities with multiple cohorts, which may influence how reproductive competitiveness is measured. Finally, unlike discrete generations models, models using OLGs can reveal how different components of fitness in a long-lived organism influence the optimal sex ratio (e.g., how important are age of first reproduction and adult life span?).

In this article, we develop a model of optimal sex ratios as a function of maternal condition in a population with OLGs. The main objective of this exercise is to examine whether sex ratio predictions from discrete generations models generalize to OLGs. In addition, we examine how fitness components of long-lived offspring interact to shape optimal sex ratios and what this tells us about how to measure offspring fitness in empirical research on longlived organisms.

\section{Maternal Condition and Sex Allocation in a Population with Discrete Generations}

We first consider a model of conditional sex ratios in a population with discrete generations for comparison with an OLG model. We use a rare-gene model to find the optimal proportion of offspring that are sons for poor $\left(r_{1}\right)$ and good $\left(r_{2}\right)$ mothers. A proportion, $h$, of females are in poor condition, and $1-h$ are in good condition. Clutch size is $b$, and the mothers in good condition provide a survival advantage to both sexes of their offspring, with the advantage to sons being greater (fig. 1A). This creates a gain asymmetry between sons and daughters, with sons gaining more from good mothers than do daughters. The first-year survival rates of sons and daughters produced by mothers in poor condition are $S_{\mathrm{m}}$ and $S_{\mathrm{f}}$, respectively. The survival rates of sons and daughters produced by mothers in good condition are $S_{\mathrm{m}} \beta_{\mathrm{m}}$ and $S_{\mathrm{f}} \beta_{\mathrm{f}}$, respectively.

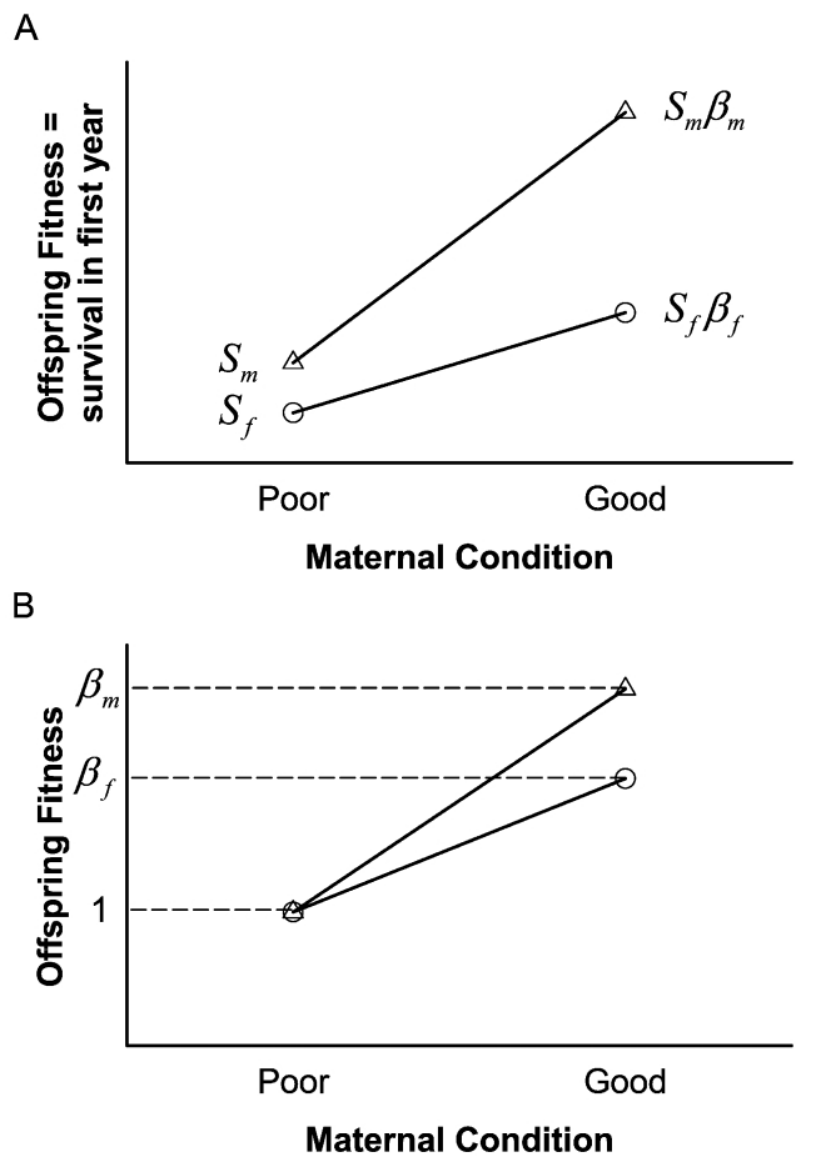

Figure 1: Maternal condition effects on offspring fitness. A, Juvenile survival of male (triangles) and female (circles) offspring from poor and good mothers. $B$, Offspring fitness standardized to 1 for offspring born in poor condition. Sons gain a greater advantage than daughters by being born to a mother in good condition. 
A

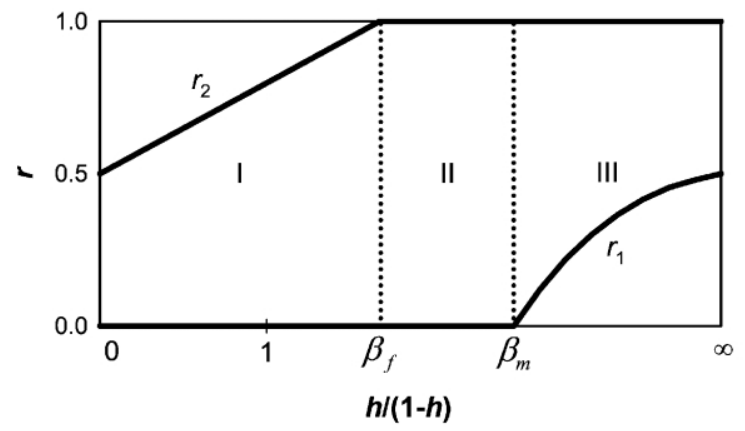

B $\beta_{f} \varphi \psi_{f}=\frac{\text { Juvenile Survival } \times \text { Fertility x Lifespan (Good daughter) }}{\text { Juvenile Survival x Fertility x Lifespan (Poor daughter) }}=\frac{R_{0}(\text { Good })}{R_{0}(\text { Poor })}$

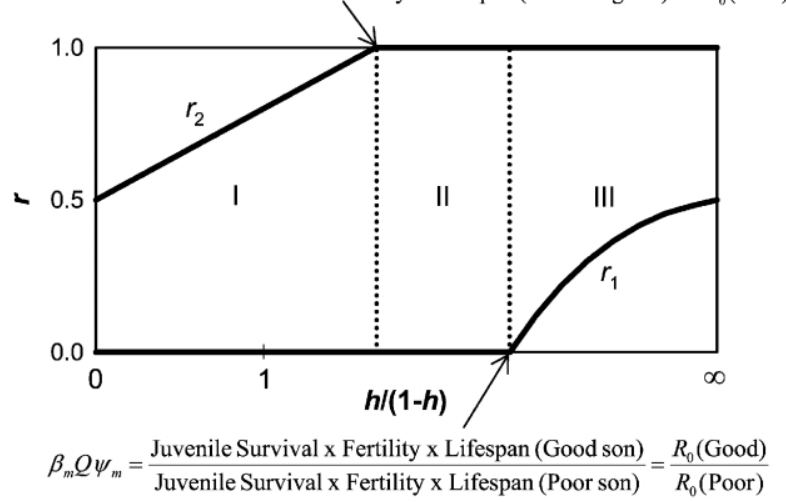

Figure 2: Optimal sex ratios (proportion of offspring that are sons) for poor $\left(r_{1}\right)$ and good $\left(r_{2}\right)$ mothers in three regions of $h /(1-h)$ space. $A$, Predictions from the discrete generations model. $B$, Predictions from the overlapping generations model. The optimal sex ratio depends on the within-sex ratio of offspring lifetime fitness $\left(R_{0}\right)$ from good and poor mothers.

Therefore, $\beta_{\mathrm{m}}$ and $\beta_{\mathrm{f}}$ represent the relative survival advantages (the ratio) of offspring born to mothers in good condition compared with those born to mothers of poor condition (here, $\beta_{\mathrm{m}}>\beta_{\mathrm{f}}>1$ ). Maternal condition has no influence on the condition in which their daughters reproduce (this is again assigned at random as $h$ and $1-$ h).

The model is presented in appendix A and is similar to sex ratio models in other condition-dependent systems (Charnov et al. 1978; Charnov 1979; Charnov and Dawson 1989). The final fitness equation (A1) and the sex ratio solutions (fig. $2 A$; table A1, eqq. [A4a], [A4b]) feature the survival advantages $\left(\beta_{\mathrm{m}}\right.$ and $\left.\beta_{\mathrm{f}}\right)$ but not the survival rates themselves $\left(S_{\mathrm{m}}\right.$ and $\left.S_{\mathrm{f}}\right)$. It is the dimensionless ratios $\left(\beta_{\mathrm{m}}\right.$ and $\beta_{\mathrm{f}}$ ) that determine fitness (fig. $1 B$ ).

The solutions reveal that both $r_{1}$ and $r_{2}$ cannot be optimized simultaneously as long as $\beta_{\mathrm{m}} \neq \beta_{\mathrm{f}}$, and one or both sex ratios must always be at a boundary ( 0 or 1 ; table
A1). The sex ratio functions $\left(r_{2}=(1 / 2)+(1 / 2)[h /(1-\right.$ h) $]\left(1 / \beta_{\mathrm{f}}\right), \quad r_{1}=(1 / 2)-(1 / 2)[(1-h) / h] \beta_{\mathrm{m}} ;$ table A1, eqq. $[\mathrm{A} 4 \mathrm{a}],[\mathrm{A} 4 \mathrm{~b}])$ depend on the ratio of females in poor condition to females in good condition $(h /(1-h)$; fig. $2 A)$, with three separate regions. First, when females in poor condition are rare, they produce only daughters, while good females produce a male-biased sex ratio. Second, when good females are rare, they produce only sons, while poor females produce a sex ratio $<0.5$. Third, at intermediate proportions, good females produce only sons, while poor females produce only daughters. This pattern in sex ratio biases is predicted as long as $\beta_{\mathrm{m}}>\beta_{\mathrm{f}}$, and the cut points between the three regions are determined by $\beta_{\mathrm{m}}$ and $\beta_{\mathrm{f}}$.

\section{Maternal Condition and Sex Allocation with OLGs}

We extend these analyses of conditional sex allocation to populations with OLGs to examine whether OLGs per se alter the model predictions. As before, $h$ proportion of females are in poor condition and produce a sex ratio of $r_{1}$, whereas $1-h$ proportion of females are in good condition and produce a sex ratio of $r_{2}$. This random assignment of breeding condition assumes that a female's birth condition does not affect the birth condition of her offspring. The age of first reproduction is 1 year, and clutch size is $b$. In this model, we include additional effects of maternal condition on offspring fitness components (table 1). The fecundity of a female is associated with her birth condition and not her current reproductive condition.

To explore maternal effects on sex allocation in populations with OLGs, we develop a population genetics model (app. B). We first consider the projection through time of a background population (wild-type, aa), with population dynamic equations for all females and males born under poor and good conditions separately (eqq. [B1]). We then introduce a rare, dominant mutant $(A a)$ into this background population and describe the population dynamics of the same life-history states (eqq. [B3]). The population dynamic equations provide a mutant fitness measure, $\lambda_{2}$ (the per-unit time increase of the mutant gene in a non-

Table 1: Fitness components for the overlapping generations model

\begin{tabular}{lccc}
\hline $\begin{array}{l}\text { Offspring sex and } \\
\text { birth condition }\end{array}$ & $\begin{array}{c}\text { First-year } \\
\text { survival }\end{array}$ & $\begin{array}{c}\text { Fertility or } \\
\text { fecundity }\end{array}$ & $\begin{array}{c}\text { Annual adult } \\
\text { survival }\end{array}$ \\
\hline Male: & $S_{\mathrm{m}} \beta_{\mathrm{m}}$ & $Q$ & $p_{\mathrm{m}} \gamma_{\mathrm{m}}$ \\
$\quad$ Good & $S_{\mathrm{m}}$ & 1 & $p_{\mathrm{m}}$ \\
Poor & & & \\
Female: & $S_{\mathrm{f}} \beta_{\mathrm{f}}$ & $b \varphi$ & $p_{\mathrm{f}} \gamma_{\mathrm{f}}$ \\
Good & $S_{\mathrm{f}}$ & $b$ & $p_{\mathrm{f}}$ \\
Poor & &
\end{tabular}


growing background population), which is used to find the optimal $r_{1}$ and $r_{2}$. As with the previous model, we find that both $r_{1}$ and $r_{2}$ cannot simultaneously have internal optima. The optimal sex ratios again depend on the ratio of the proportion of females in poor condition to the proportion of females in good condition, resulting in three regions of $h /(1-h)$ (table 2 ; fig. $2 B$ ).

The solutions (table 2, eqq. [1a], [1b]) are essentially unchanged from those for the rare-gene, discrete generations model (cf. table A1, eqq. [A4a], [A4b]). Both solutions reflect the addition of new advantages to sons and daughters born to mothers in good condition: $\beta_{\mathrm{m}}$ and $\beta_{\mathrm{f}}$ are the familiar first-year survival advantages, $Q$ and $\varphi$ are the fertility and fecundity advantages, and $\psi_{\mathrm{m}}$ and $\psi_{\mathrm{f}}$ are adult life span advantages determined by annual adult survival advantages $\left(\gamma_{\mathrm{m}}\right.$ and $\left.\gamma_{\mathrm{f}}\right)$. The predicted optimal sex ratios depend on the product of these advantages in fitness components (juvenile survival advantage $\times$ adult life span advantage $\times$ fertility advantage). That is, the cut points for $r_{1}$ and $r_{2}$ (boundaries between regions I, II, and III) are redefined to represent the lifetime fitness advantage of being born to a mother in good condition (fig. $2 \mathrm{~B}$; ratio of juvenile survival $\times$ fecundity $\times$ adult life span for good to poor offspring). As long as the combined fitness advantage to sons is greater than the advantage to daughters $\left(\beta_{\mathrm{m}} Q \psi_{\mathrm{m}}>\beta_{\mathrm{f}} \varphi \psi_{\mathrm{f}}\right)$, mothers in good condition will produce male-biased sex ratios, and mothers in poor condition will produce female-biased sex ratios. This model demonstrates that the presence of OLGs has no effect on sex allocation; it merely redefines how we measure fitness (fig. 2B). Now fitness is lifetime fitness.

\section{Additions to the Model: General Rules for OLGs}

We made two modifications to the model to explore its application to other biological situations (see app. B for more details). First, we changed the age of first reproduction (AFR) to 2 years for both males and females. The sex ratio predictions of a model with $\mathrm{AFR}=2$ are iden- tical to those for AFR $=1$. In general, we can consider an organism that reaches sexual maturity many years after birth, where annual survival each year prior to sexual maturity may differ between good and poor offspring. In this case, the total immature survival advantage would simply be the product of the yearly survival advantages (e.g., $\left.\beta_{\mathrm{f} 1} \beta_{\mathrm{f} 2} \beta_{\mathrm{f} 3}\right)$. Notably, a conditional advantage seen solely in first-year survival $\left(\beta_{\mathrm{f} 1}\right.$ or $\left.\beta_{\mathrm{m} 1}\right)$ would still be measured in the total juvenile survival advantage. This demonstrates the following important result: for long-lived organisms that have OLGs and that do not reproduce until many years after birth, a sex-differential conditional advantage in first-year survival alone is sufficient to predict biased sex ratios (i.e., Q, $\psi_{\mathrm{m}}, \varphi$, and $\psi_{\mathrm{f}}=1$ ). In other words, maternal conditional effects need not be maintained until adulthood for biased sex ratios to be adaptive.

Second, we examined the situation where females breeding in good condition show an increase in fecundity compared with females in poor condition. Females in good condition were given a fecundity of $b \varphi$, where $\varphi$ is the ratio of good female fecundity to poor female fecundity $(\varphi>1)$. Breeding condition is still assigned at random. This fecundity advantage to females reproducing in good condition does not affect the solutions. However, it does increase the number of sons and daughters born in good condition and redefines the region boundaries in a manner equivalent to rescaling the $h /(1-h)$ axis by a factor of $\varphi^{-1}$.

Although we have not explored arbitrary AFR or agespecific demography or fertility, the models lead us to anticipate several generalities associated with sex ratios in nongrowing populations with OLGs. Our models demonstrate that the sex ratio solutions depend on advantages in offspring lifetime fitness (within each sex). This lifetime fitness rule is equivalent to using $R_{0}$ as the fitness measure (Charnov 1997) and should hold regardless of the particular model demography, as long as condition is assigned randomly (fig. 2B; Leimar 1996). The direction of sex ratio biases should always be predicted by the ratio of son versus

Table 2: Maternal condition and optimal sex ratios in a population with overlapping generations

\begin{tabular}{llcc}
\hline Derivatives & \multicolumn{1}{c}{$\begin{array}{c}\text { Parameter space } \\
\text { regions }\end{array}$} & $r_{1}$ & $r_{2}$ \\
\hline I. $\frac{d \lambda_{2}}{d \hat{r}_{1}}<0, \frac{d \lambda_{2}}{d \hat{r}_{2}}=0$ & $\frac{h}{1-h}<\beta_{\mathrm{f}} \varphi \psi_{\mathrm{f}}$ & 0 & $\frac{1}{2}+\frac{1}{2} \frac{h}{(1-h)} \frac{1}{\beta_{f} \varphi \psi_{f}}$ (eq. [1a]) \\
II. $\frac{d \lambda_{2}}{d \hat{r}_{1}}<0, \frac{d \lambda_{2}}{d \hat{r}_{2}}>0$ & $\beta_{\mathrm{f}} \varphi \psi_{\mathrm{f}} \leq \frac{h}{1-h} \leq \beta_{\mathrm{m}} \mathrm{Q} \psi_{\mathrm{m}}$ & 0 & 1 \\
III. $\frac{d \lambda_{2}}{d \hat{r}_{1}}=0, \frac{d \lambda_{2}}{d \hat{r}_{2}}>0$ & $\beta_{\mathrm{m}} \mathrm{Q} \psi_{\mathrm{m}}<\frac{h}{1-h}$ & $\frac{1}{2}-\frac{1}{2} \frac{(1-h)}{h} \beta_{\mathrm{m}} Q \psi_{\mathrm{m}}$ (eq. [1b]) & 1 \\
\hline
\end{tabular}

Note: $\psi_{\mathrm{m}}$ is the ratio of expected adult life span of a son born in good condition $\left(E_{\mathrm{mg}}\right)$ to the expected adult life span of a son born in poor condition $\left(E_{\mathrm{mp}}\right), E_{\mathrm{mg}} / E_{\mathrm{mp}} ; E_{\mathrm{mg}}=1 /\left(1-p_{\mathrm{m}} \gamma_{\mathrm{m}}\right)$ and $E_{\mathrm{mp}}=1 /\left(1-\mathrm{p}_{\mathrm{m}}\right) . \psi_{\mathrm{f}}$ is the ratio of expected adult life span of a daughter born in good condition $\left(E_{\mathrm{fg}}\right)$ to the expected adult life span of a daughter born in poor condition $\left(E_{\mathrm{ff}}\right), E_{\mathrm{fg}} / E_{\mathrm{fp}}$; $E_{\mathrm{fg}}=1 /\left(1-p_{\mathrm{f}} \gamma_{\mathrm{f}}\right)$ and $E_{\mathrm{fp}}=1 /\left(1-p_{\mathrm{f}}\right)$. 
daughter lifetime fitness advantages. In addition, conditional effects that impact both sexes equally (e.g., increased maternal fecundity) have little or no influence on the sex ratio predictions.

\section{Discussion and Conclusions}

We have developed a model of optimal sex allocation when maternal condition varies for populations with OLGs. It takes into account a number of biological scenarios, and its flexibility (e.g., altering various aspects of offspring fitness and population demography) should be of great use for future theoretical extensions. The life-history aspects incorporated in our models include maternal effects on all components of offspring lifetime fitness $\left(R_{0}\right)$, delayed age of first reproduction, and condition-based fecundity benefits. Our model has demonstrated that the presence of OLGs per se has no effect on the sex ratio predictions. Of course, this generalization must be accompanied by the caveat that many social and reproductive characteristics often thought to impact sex ratios operate only when generations overlap (e.g., daughters may remain near and interfere with their mother's future reproduction).

The model we have developed in this article also advances our views on how fitness effects in offspring shape optimal sex ratios. Similar to other sex ratio models, we found that the optimal sex ratios depend on the relative within-sex advantages of maternal condition and that absolute differences in offspring fitness between the sexes did not matter (Charnov 1979). In addition, offspring juvenile survival, adult life span, and fertility combine to redefine offspring fitness as lifetime fitness or $R_{0}$. When one offspring sex gains more in lifetime fitness from being born to good-condition mothers (e.g., males: $\beta_{\mathrm{m}} Q \psi_{\mathrm{m}}>\beta_{\mathrm{f}} \varphi \psi_{\mathrm{f}}$ ), that sex will be overrepresented in the offspring of those mothers. The importance of lifetime fitness has previously been argued for maternal-condition-based sex ratios by Leimar (1996). Here, we show that offspring lifetime fitness is the product of three measurable fitness components (juvenile survival $\times$ adult life span $\times$ annual fertility; Charnov 1997).

This lifetime fitness rule indicates which aspects of offspring phenotype and life history should be emphasized in empirical research on sex ratios. In the conceptual model of Trivers and Willard (1973), maternal condition affected the reproductive success of sons more than that of daughters (assumption 3). This is captured in our model by the fertility and fecundity variables $(Q$ and $\varphi)$. This emphasis on adult reproductive success (fertility and fecundity) has led to much attention being placed on the second T-W assumption that maternal effects are maintained until adulthood. Here, we've confirmed that neither of these assumptions needs to be met in order for condition-dependent sex ratios to be adaptive. Fertility advantages represent only one of the three fitness components in the lifetime fitness of each sex of offspring. The other two are juvenile survival and adult life span. If maternal condition influences only offspring survival from weaning to sexual maturity, this is potentially sufficient to lead to biased sex ratios. In addition, if any of the lifetime fitness components combine to provide a sex-differential advantage with condition, our model predicts conditiondependent biased sex ratios.

Our model also illustrates how opposing advantages to offspring fitness components might select for unbiased sex ratios based on maternal condition. For example, sons may gain more in fertility from maternal condition (i.e., T-W hypothesis), but if daughters gain equally more in juvenile survival, this could negate a benefit for biased sex ratios. Therefore, empirical research on conditional sex ratios (including T-W) should attempt to measure all three components of lifetime fitness (juvenile survival, adult life span, and fertility) rather than focusing on fertility alone (for a similar argument, see Leimar 1996). This is difficult in practice but is essential for an accurate prediction of offspring sex ratios.

Finally, we do not wish to leave the impression that agestructured life histories never show the potential to select for facultative sex ratios due to age structure itself, only that a maternal condition effect may not change with OLGs. At least two age-structured facultative effects have been studied. Short-lived organisms in seasonal environments with small overlap in generations (i.e., across-season age structure) may have adaptive seasonal shifts in sex ratio (Werren and Charnov 1978). These shifts are known for several species (Seger 1983; Conover and Heins 1987) and may occur in mammals (e.g., McShea and Madison 1986). As a second example, long life in mammals is often correlated with a litter size of 1 . If a male offspring imposes a different mortality rate on the mother than a daughter does, a shift in offspring sex is predicted with the mother's age such that the sex imposing the lower mortality should be born to younger mothers (Charnov 1982, p. 96). This is an age-structured prediction even for mothers of the same quality because mothers showing the age shift will increase their own life span. This effect seems not to be consistently observed in mammals (Hewison et al. 2002).

We imagine some logical extensions of the model that take into account further potential biological complications. Some of these extensions might help elucidate the inconsistent sex ratios of mammals. First, adding an interaction between maternal condition and a daughter's adult reproductive condition may alone create a relative advantage for daughters (Leimar 1996), although it remains unclear as to when this would overcome a son's relative advantage and lead to production of daughters by 
mothers in good condition. Second, we could include small clutch size effects and sex-specific quality versus offspring size curves to examine whether a mother with adequate resources should have one high-quality son or two average daughters (Williams 1979). Third, there may be costs or constraints of sex ratio manipulation (e.g., to fecundity; Pen and Weissing 2002). If the sex ratio itself can be moved only in the region of, say, 0.35-0.65, we expect that boundary sex ratios would be at the allowable extremes. Finally, multiple forces (e.g., conditional, social) may impact offspring fitness in mammals and may interact to accentuate or cancel the benefits of sex ratio biases (Cockburn et al. 2002). For example, in some mammals, competition with daughters may be important in shaping sex ratios at high population density or in young mothers (local resource competition; Clark 1978), while maternal condition becomes more important for sex ratios at low density or in older mothers (e.g., van Schaik and Hrdy 1991; Kruuk et al. 1999; Isaac et al. 2005). The OLG model we have presented in this article can provide the foundation for models that examine these interacting effects and, potentially, shed light on the diversity of natural sex ratios observed in mammals.

\section{Acknowledgments}

L.E.S. was supported by a National Science Foundation (NSF) Graduate Research Fellowship, and J.G.B. was supported by an NSF Biocomplexity Grant (DEB-0083422). J. H. Brown, J. J. Bull, A. Kodric-Brown, and an anonymous reviewer provided comments on a previous draft of the manuscript.

\section{APPENDIX A}

\section{Discrete Generations}

Our model follows closely the original argument introduced by Shaw and Mohler (1953). We count the contribution of genes to grandchildren by a single mutant mother who produces sex ratios of $\hat{r}_{1}$ and $\hat{r}_{2}$ in a population of wildtype mothers producing $r_{1}$ and $r_{2}$. Suppose $N$ wild-type mothers (plus the mutant) each produce sons and daughters. Subsequently, the offspring reproduce, the population of new adults produces $K$ fertilized zygotes (grandchildren), and $K$ grandchildren have $2 K$ haploid chromosome sets (HCS), $K$ of which come from males and $K$ of which come from females. The mutant mother's fitness $(W)$ is the proportion of HCS that came from her offspring.

If $N$ is large, the mutant mother's contribution to grandchildren through sons and daughters is

$$
\begin{aligned}
& K \frac{h \hat{r}_{1} b S_{\mathrm{m}}+(1-h) \hat{r}_{2} b S_{\mathrm{m}} \beta_{\mathrm{m}}}{N\left[h r_{1} b S_{\mathrm{m}}+(1-h) r_{2} b S_{\mathrm{m}} \beta_{\mathrm{m}}\right]}, \\
& K \frac{h\left(1-\hat{r}_{1}\right) b S_{\mathrm{f}}+(1-h)\left(1-\hat{r}_{2}\right) b S_{\mathrm{f}} \beta_{\mathrm{f}}}{N\left[h\left(1-r_{1}\right) b S_{\mathrm{f}}+(1-h)\left(1-r_{2}\right) b S_{\mathrm{f}} \beta_{\mathrm{f}}\right]} .
\end{aligned}
$$

The total genetic contribution of the mutant mother to grandchildren is then

$$
W=\left[\frac{h \hat{r}_{1}+(1-h) \hat{r}_{2} \beta_{\mathrm{m}}}{h r_{1}+(1-h) r_{2} \beta_{\mathrm{m}}}+\frac{h\left(1-\hat{r}_{1}\right)+(1-h)\left(1-\hat{r}_{2}\right) \beta_{\mathrm{f}}}{h\left(1-r_{1}\right)+(1-h)\left(1-r_{2}\right) \beta_{\mathrm{f}}}\right] \frac{K}{N} .
$$

Without loss of generality, we also set $K / N=1$.

Because a wild-type organism has $W=2$, a mutant will invade the population if it has $W>2$. The sex ratios $r_{1}$ and $r_{2}$ are evolutionarily stable if there are no mutant values $\left(\hat{r}_{1}, \hat{r}_{2}\right)$ that make $W>2$. At the evolutionarily stable strategy (ESS) conditions: (1) $W$ is maximized with respect to $\hat{r}_{1}$ and $\hat{r}_{2},(2) \hat{r}_{1}=r_{1}$, and $\hat{r}_{2}=r_{2}$, and (3) $W=2$. To find the ESS, we consider the derivatives $d W / d \hat{r}_{1}$ and $d W / d \hat{r}_{2}$, when $\hat{r}_{1}=r_{1}$ and $\hat{r}_{2}=r_{2}$. The derivatives are

$$
\begin{aligned}
& \frac{d W}{d \hat{r}_{1}}=\frac{h}{h r_{1}+(1-h) r_{2} \beta_{\mathrm{m}}}+\frac{-h}{h\left(1-r_{1}\right)+(1-h)\left(1-r_{2}\right) \beta_{\mathrm{f}}}, \\
& \frac{d W}{d \hat{r}_{2}}=\frac{(1-h) \beta_{\mathrm{m}}}{h r_{1}+(1-h) r_{2} \beta_{\mathrm{m}}}+\frac{-(1-h) \beta_{\mathrm{f}}}{h\left(1-r_{1}\right)+(1-h)\left(1-r_{2}\right) \beta_{\mathrm{f}}} .
\end{aligned}
$$


If we set $M=h r_{1}+(1-h) r_{2} \beta_{\mathrm{m}}$ and $F=h\left(1-r_{1}\right)+(1-h)\left(1-r_{2}\right) \beta_{\mathrm{f}}$, we find

$$
\begin{aligned}
& \frac{d W}{d \hat{r}_{1}}=\frac{1}{M}-\frac{1}{F} \\
& \frac{d W}{d \hat{r}_{2}}=\frac{\beta_{\mathrm{m}}}{M}-\frac{\beta_{\mathrm{f}}}{F} .
\end{aligned}
$$

With $\beta_{\mathrm{m}}>\beta_{\mathrm{f}}$, these two derivatives cannot simultaneously equal 0 . Consideration of equations (A3) reveals that there are three possible situations (see table A1; fig. $2 A$ ).

Table A1: Maternal condition and optimal sex ratios in a population with discrete generations

\begin{tabular}{llcc}
\hline Derivatives & \multicolumn{1}{c}{$\begin{array}{c}\text { Parameter space } \\
\text { regions }\end{array}$} & $r_{1}$ & $r_{2}$ \\
\hline I. $\frac{d W}{d \hat{r}_{1}}<0, \frac{d W}{d \hat{r}_{2}}=0$ & $\frac{h}{1-h}<\beta_{\mathrm{f}}$ & 0 & $\frac{1}{2}+\frac{1}{2} \frac{h}{(1-h)} \frac{1}{\beta_{\mathrm{f}}}$ (eq. [A4a]) \\
II. $\frac{d W}{d \hat{r}_{1}}<0, \frac{d W}{d \hat{r}_{2}}>0$ & $\beta_{\mathrm{f}} \leq \frac{h}{1-h} \leq \beta_{\mathrm{m}}$ & 0 & 1 \\
III. $\frac{d W}{d \hat{r}_{1}}=0, \frac{d W}{d \hat{r}_{2}}>0$ & $\beta_{\mathrm{m}}<\frac{h}{1-h}$ & $\frac{1}{2}-\frac{1}{2} \frac{(1-h)}{h} \beta_{\mathrm{m}}$ (eq. [A4b]) & 1 \\
\hline
\end{tabular}

\section{APPENDIX B}

\section{Overlapping Generations}

To examine condition-dependent sex ratios in a population with overlapping generations, we first describe the background (wild-type, $a a$ ) population using population dynamic equations. We count the number of females born under poor condition $\left(n_{\mathrm{p}}(t)\right)$, females born under good condition $\left(n_{\mathrm{g}}(t)\right)$, males born under poor condition $\left(n_{\mathrm{p}}^{*}(t)\right)$, and males born under good condition $\left(n_{\mathrm{g}}^{*}(t)\right)$. In stable-age distribution,

$$
\begin{aligned}
& n_{\mathrm{p}}(t+1)=n_{\mathrm{p}}(t) p_{\mathrm{f}}+n_{\mathrm{p}}(t) h\left(1-r_{1}\right) b S_{\mathrm{f}}+n_{\mathrm{g}}(t) h\left(1-r_{1}\right) b \varphi S_{\mathrm{f}}=\lambda_{1} n_{\mathrm{p}}(t), \\
& n_{\mathrm{g}}(t+1)=n_{\mathrm{g}}(t) p_{\mathrm{f}} \gamma_{\mathrm{f}}+n_{\mathrm{p}}(t)(1-h)\left(1-r_{2}\right) b S_{\mathrm{f}} \beta_{\mathrm{f}}+n_{\mathrm{g}}(t)(1-h)\left(1-r_{2}\right) b \varphi S_{\mathrm{f}} \beta_{\mathrm{f}}=\lambda_{1} n_{\mathrm{g}}(t), \\
& n_{\mathrm{p}}^{*}(t+1)=n_{\mathrm{p}}^{*}(t) p_{\mathrm{m}}+n_{\mathrm{p}}(t) h r_{1} b S_{\mathrm{m}}+n_{\mathrm{g}}(t) h r_{1} b \varphi S_{\mathrm{m}}=\lambda_{1} n_{\mathrm{p}}^{*}(t), \\
& n_{\mathrm{g}}^{*}(t+1)=n_{\mathrm{g}}^{*}(t) p_{\mathrm{m}} \gamma_{\mathrm{m}}+n_{\mathrm{p}}(t)(1-h) r_{2} b S_{\mathrm{m}} \beta_{\mathrm{m}}+n_{\mathrm{g}}(t)(1-h) r_{2} b \varphi S_{\mathrm{m}} \beta_{\mathrm{m}}=\lambda_{1} n_{\mathrm{g}}^{*}(t),
\end{aligned}
$$

where $\lambda_{1}$ is the dominant eigenvalue of the transition matrix. The population is growing when $\lambda_{1}>1$ and stable when $\lambda_{1}=1$. In order to find $\lambda_{1}$, we find the characteristic equation of the transition matrix. We assume a nongrowing background population $\left(\lambda_{1}=1\right)$, and so we must also assume that at least one variable in $\lambda_{1}$ is density dependent. We propose that $S_{\mathrm{f}}$ is the most likely variable to carry the density dependence and solve accordingly:

$$
S_{\mathrm{f}}=\frac{1-p_{\mathrm{f}}}{b\left[h\left(1-r_{1}\right)+(1-h)\left(1-r_{2}\right) \beta_{\mathrm{f}} \varphi \psi_{\mathrm{f}}\right]},
$$

where $\psi_{\mathrm{f}}=\left(1-p_{\mathrm{f}}\right) /\left(1-p_{\mathrm{f}} \gamma_{\mathrm{f}}\right)$. In practice, density dependence could be anywhere in $b S_{\mathrm{f}}$.

Now we consider a mutant $(A a)$ that is introduced into the population. When the mutant is rare, all mutants mate only with wild-type individuals $(A a \times a a)$ so that half of all offspring produced by mutants are themselves mutants. Mutant offspring arise through mutant females mating with wild-type males and mutant males mating with wild-type females. The mutant produces offspring sex ratios $\hat{r}_{1}$ and $\hat{r}_{2}$. As with the background population, we can write out the growth equations for the mutants (a circumflex denotes mutant parameters) as follows: 


$$
\begin{aligned}
\hat{n}_{\mathrm{p}}(t+1)= & \hat{n}_{\mathrm{p}}(t) p_{\mathrm{f}}+\left[\hat{n}_{\mathrm{p}}(t)+\hat{n}_{\mathrm{g}}(t) \varphi\right]\left[\frac{1}{2} h\left(1-\hat{r}_{1}\right) b S_{\mathrm{f}}\right] \\
& +\left[n_{\mathrm{p}}(t)+n_{\mathrm{g}}(t) \varphi\right]\left[\frac{1}{2} h\left(1-r_{1}\right) b S_{\mathrm{f}}\right]\left[\frac{\hat{n}_{\mathrm{p}}^{*}(t)+\hat{n}_{\mathrm{g}}^{*}(t) Q}{\left.n_{\mathrm{p}}^{*}(t)+n_{\mathrm{g}}^{*}(t) Q\right]}\right. \\
\hat{n}_{\mathrm{g}}(t+1)= & \hat{n}_{\mathrm{g}}(t) p \gamma_{\mathrm{f}}+\left[\hat{n}_{\mathrm{p}}(t)+\hat{n}_{\mathrm{g}}(t) \varphi\right]\left[\frac{1}{2}(1-h)\left(1-\hat{r}_{2}\right) b S_{\mathrm{f}} \beta_{\mathrm{f}}\right] \\
& +\left[n_{\mathrm{p}}(t)+n_{\mathrm{g}}(t) \varphi\right]\left[\frac{1}{2}(1-h)\left(1-r_{2}\right) b S_{\mathrm{f}} \beta_{\mathrm{f}}\right]\left[\frac{\left.\hat{n}_{\mathrm{p}}^{*}(t)+\hat{n}_{\mathrm{g}}^{*}(t) Q\right]}{\left.n_{\mathrm{p}}^{*}(t)+n_{\mathrm{g}}^{*}(t) Q\right]}\right. \\
\hat{n}_{\mathrm{p}}^{*}(t+1)= & \hat{n}_{\mathrm{p}}^{*}(t) p_{\mathrm{m}}+\left[\hat{n}_{\mathrm{p}}(t)+\hat{n}_{\mathrm{g}}(t) \varphi\right]\left(\frac{1}{2} h \hat{r}_{1} b S_{\mathrm{m}}\right) \\
& +\left[n_{\mathrm{p}}(t)+n_{\mathrm{g}}(t) \varphi\right]\left(\frac{1}{2} h r_{1} b S_{\mathrm{m}}\right)\left[\frac{\hat{n}_{\mathrm{p}}^{*}(t)+\hat{n}_{\mathrm{g}}^{*}(t) Q}{\left.n_{\mathrm{p}}^{*}(t)+n_{\mathrm{g}}^{*}(t) Q\right]}\right. \\
& +\left[n_{\mathrm{p}}(t)+n_{\mathrm{g}}(t) \varphi\right]\left[\frac{1}{2}(1-h) r_{2} b S_{\mathrm{m}} \beta_{\mathrm{m}}\right]\left[\frac{\hat{n}_{\mathrm{p}}^{*}(t)+\hat{n}_{\mathrm{g}}^{*}(t) Q}{\left.n_{\mathrm{p}}^{*}(t)+n_{\mathrm{g}}^{*}(t) Q\right]}\right. \\
\hat{n}_{\mathrm{g}}^{*}(t+1)= & \hat{n}_{\mathrm{g}}^{*}(t) p_{\mathrm{m}} \gamma_{\mathrm{m}}+\left[\hat{n}_{\mathrm{p}}(t)+\hat{n}_{\mathrm{g}}(t) \varphi\right]\left[\frac{1}{2}(1-h) \hat{r}_{2} b S_{\mathrm{m}} \beta_{\mathrm{m}}\right] \\
&
\end{aligned}
$$

For the mutant to invade, the dominant eigenvalue of the mutant population matrix, $\lambda_{2}$, must be greater than that of the background population $\left(\lambda_{1}\right)$. Because we assume a nongrowing background population $\left(\lambda_{1}=1\right)$, $\lambda_{2}$ must be $>$ 1. The sex ratios $r_{1}$ and $r_{2}$ are evolutionarily stable if there are no mutant values $\left(\hat{r}_{1}, \hat{r}_{2}\right)$ that make $\lambda_{2}>\lambda_{1}$. At the ESS conditions, (1) $\lambda_{2}$ is maximized with respect to $\hat{r}_{1}$ and $\hat{r}_{2}$, (2) $\hat{r}_{1}=r_{1}$ and $\hat{r}_{2}=r_{2}$, and $(3) \lambda_{2}=1$.

To find $\lambda_{2}$, we must find the characteristic equation of the mutant matrix $\left\{\mathbf{A}_{2}\right\}$. Equations (B3) can be written in terms of $R$, the adult sex ratio weighted by the fertility advantage of good males and females:

$$
R=\frac{n_{\mathrm{p}}(t)+n_{\mathrm{g}}(t) \varphi}{n_{\mathrm{p}}^{*}(t)+n_{\mathrm{g}}^{*}(t) Q} .
$$

The mutant matrix, $\left\{\mathbf{A}_{2}\right\}$, is then

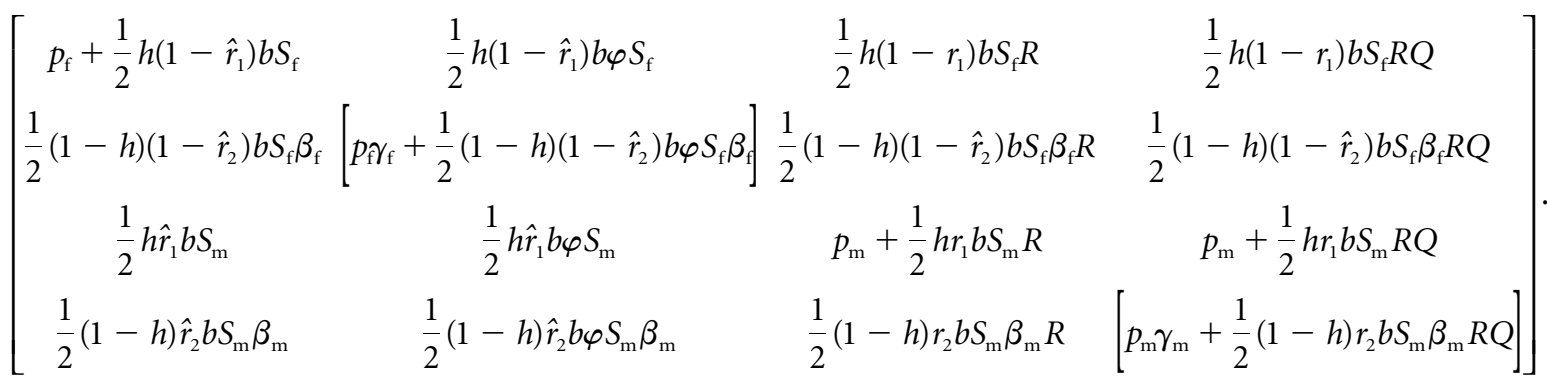

Assuming the background population is in stable-age distribution, we use equations (B1c) and (B1d) to find

$$
R=\left[\frac{h r_{1} b S_{\mathrm{m}}}{1-p_{\mathrm{m}}}+\frac{(1-h) r_{2} b S_{\mathrm{m}} \beta_{\mathrm{m}} Q}{1-p_{\mathrm{m}} \gamma_{\mathrm{m}}}\right]^{-1}
$$

We also substitute equation (B2) into the matrix for $S_{\mathrm{f}}$ so that the same density dependence applies to the mutant. 
From the characteristic equation of $\left\{\mathbf{A}_{2}\right\}$, we find $\lambda_{2}$. To find the ESS, we consider the derivatives $d \lambda_{2} / d \hat{r}_{1}$ and $d \lambda_{2} / d \hat{r}_{2}$, when $\hat{r}_{1}=r_{1}$ and $\hat{r}_{2}=r_{2}$, and set these equal to 0 . We performed the following calculations (see app. C in the online edition of the American Naturalist): (1) Use the background transition matrix to solve for $S_{\mathrm{f}}$. (2) Calculate the characteristic equation of $\left\{\mathbf{A}_{2}\right\}$. (3) Take the derivative of $\lambda_{2}$, with respect to $\hat{r}_{1}$ and $\hat{r}_{2}$. (4) Apply ESS conditions: $\hat{r}_{1}=r_{1}, \hat{r}_{2}=r_{2}$, and $\lambda_{2}=\lambda_{1}=1$. (5) Solve for $r_{1}$ and $r_{2}$ when $d \lambda_{2} / d \hat{r}_{1}=0$ and $d \lambda_{2} / d \hat{r}_{2}=0$, respectively. (6) Confirm that both derivatives cannot be optimized simultaneously (cannot equal 0 simultaneously). (7) Solve for $r_{1}$ with $r_{2}=1$. Solve for $r_{2}$ with $r_{1}=0$. These are the final solutions. (8) Confirm the assumptions in step 7 that if $r_{1}$ has an internal optimum, then $r_{2}=1$ and if $r_{2}$ has an internal optimum, then $r_{1}=0\left(\right.$ for $\left.\beta_{\mathrm{m}} Q \psi_{\mathrm{m}}>\beta_{\mathrm{f}} \varphi \psi_{\mathrm{f}}\right)$.

\section{Additional Model Modifications}

Age of First Reproduction of 2 Years. To modify the model for age of first reproduction of 2 years rather than 1 year, we track (1) first-year females (daughters produced by females $\geq 2$ years old $\times$ juvenile survival from the previous year) and (2) $\geq 2$-year-old females (survival of first-year and $\geq 2$-year-old females from the previous year). Survival after the first year is equal to adult survival. We treat males in a comparable fashion. We assumed that adult survival does not differ between offspring born in different conditions, and the only advantage to offspring born in good condition is a first-year survival advantage to sons. The process of finding the ESS sex ratios follows the same steps as before.

Females Reproducing in Good Condition Have a Fecundity Advantage. To give a fecundity advantage to females reproducing in good condition, we assign a fecundity of $b \varphi$ to the $1-h$ proportion of females in good condition, where $\varphi>1$. The fecundity advantage is due only to the condition in which the female is reproducing that year and is not influenced by the condition in which she was born. Advantages to offspring from being born to a mother in good condition are seen only in juvenile survival, with $\beta_{\mathrm{m}}>\beta_{\mathrm{f}}>1$. We use one equation each to count females and males in the population. The process of finding the ESS sex ratios follows similar steps as before.

\section{Literature Cited}

Bercovitch, F. B., A. Widdig, and P. Nurnberg. 2000. Maternal investment in rhesus macaques (Macaca mulatta): reproductive costs and consequences of raising sons. Behavioral Ecology and Sociobiology 48:1-11.

Blanchard, P., M. Festa-Bianchet, J. M. Gaillard, and J. T. Jorgenson. 2005. Maternal condition and offspring sex ratio in polygynous ungulates: a case study of bighorn sheep. Behavioral Ecology 16: 274-279.

Bull, J. J. 1981. Sex ratio evolution when fitness varies. Heredity 46: 9-26.

Cameron, E. Z. 2004. Facultative adjustment of mammalian sex ratios in support of the Trivers-Willard hypothesis: evidence for a mechanism. Proceedings of the Royal Society B: Biological Sciences 271: $1723-1728$.

Cameron, E. Z., W. L. Linklater, K. J. Stafford, and C. J. Veltman. 1999. Birth sex ratios relate to mare condition at conception in Kaimanawa horses. Behavioral Ecology 10:472-475.

Charnov, E. L. 1979. The genetical evolution of patterns of sexuality: Darwinian fitness. American Naturalist 113:465-480.

. 1982. The theory of sex allocation. Princeton University Press, Princeton, NJ. Oxford.

1993. Life history invariants. Oxford University Press,

- 1997. Trade-off-invariant rules for evolutionarily stable life histories. Nature 387:393-394.

Charnov, E. L., and J. Bull. 1977. When is sex environmentally determined? Nature 266:828-830.

Charnov, E. L., and T. E. Dawson. 1989. Environmental sex deter- mination with overlapping generations. American Naturalist 134: 806-816.

Charnov, E. L., D. W. Gotshall, and J. G. Robinson. 1978. Sex ratio: adaptive response to population fluctuations in pandalid shrimp. Science 200:204-206.

Charnov, E. L., R. L. Los-den Hartogh, W. T. Jones, and J. van den Assem. 1981. Sex ratio evolution in a variable environment. Nature 289:27-33.

Clark, A. B. 1978. Sex ratio and local resource competition in a prosimian primate. Science 201:163-165.

Clutton-Brock, T. H., S. D. Albon, and F. E. Guinness. 1986. Great expectations: dominance, breeding success and offspring sex ratios in red deer. Animal Behaviour 34:460-471.

Cockburn, A., S. Legge, and M. C. Double. 2002. Sex ratios in birds and mammals: can the hypotheses be disentangled? Pages 266286 in I. C. W. Hardy, ed. Sex ratios: concepts and research methods. Cambridge University Press, Cambridge.

Conover, D. O., and S. W. Heins. 1987. Adaptive variation in environmental and genetic sex determination in a fish. Nature 326: 496-498.

de Jong, T. J., and P. G. L. Klinkhamer. 2005. Evolutionary ecology of plant reproductive strategies. Cambridge University Press, Cambridge.

Fisher, D. O. 1999. Offspring sex ratio variation in the bridled nailtail wallaby, Onychogalea fraenata. Behavioral Ecology and Sociobiology 45:411-419.

Frank, S. A. 1990. Sex allocation theory for birds and mammals. Annual Review of Ecology and Systematics 21:13-55. 
Ghiselin, M. T. 1969. The evolution of hermaphroditism among animals. Quarterly Review of Biology 44:189-208.

Hewison, A. J. M., and J. M. Gaillard. 1999. Successful sons or advantaged daughters? the Trivers-Willard model and sex-biased maternal investment in ungulates. Trends in Ecology \& Evolution 14: 229-234.

Hewison, A. J. M., J. M. Gaillard, and M. Festa-Bianchet. 2002. Maternal age is not a predominant determinant of progeny sex ratio variation in ungulates. Oikos 98:334-339.

Isaac, J. L., A. K. Krockenberger, and C. N. Johnson. 2005. Adaptive sex allocation in relation to life-history in the common brushtail possum, Trichosurus vulpecula. Journal of Animal Ecology 74:552558.

Krackow, S. 1997. Maternal investment, sex-differential prospects, and the sex ratio in wild house mice. Behavioral Ecology and Sociobiology 41:435-443.

Kruuk, L. E. B., T. H. Clutton-Brock, S. D. Albon, J. M. Pemberton, and F. E. Guinness. 1999. Population density affects sex ratio variation in red deer. Nature 399:459-461.

Leimar, O. 1996. Life-history analysis of the Trivers and Willard sexratio problem. Behavioral Ecology 7:316-325.

McShea, W. J., and D. M. Madison. 1986. Sex ratio shifts within litters of meadow voles (Microtus pennsylvanicus). Behavioral Ecology and Sociobiology 18:431-436.

Pen, I., and F. J. Weissing. 2002. Optimal sex allocation: steps towards a mechanistic theory. Pages $26-45$ in I. C. W. Hardy, ed. Sex ratios: concepts and research methods. Cambridge University Press, Cambridge.

Pen, I., F. J. Weissing, and S. Daan. 1999. Seasonal sex ratio trend in the European kestrel: an evolutionarily stable strategy analysis. American Naturalist 153:384-397.
Seger, J. 1983. Partial bivoltinism may cause alternating sex-ratio biases that favour eusociality. Nature 301:59-62.

Shaw, R. F., and J. D. Mohler. 1953. The selective significance of the sex ratio. American Naturalist 87:337-342.

Sheldon, B. C., and S. A. West. 2004. Maternal dominance, maternal condition, and offspring sex ratio in ungulate mammals. American Naturalist 163:40-54.

Trivers, R. L., and D. E. Willard. 1973. Natural selection of parental ability to vary the sex ratio of offspring. Science 179:90-92.

van Schaik, C. P., and S. B. Hrdy. 1991. Intensity of local resource competition shapes the relationship between maternal rank and sex ratios at birth in cercopithecine primates. American Naturalist 138:1555-1562.

Werren, J. H., and E. L. Charnov. 1978. Facultative sex ratios and population dynamics. Nature 272:349-350.

West, S. A., and H. C. J. Godfray. 1997. Sex ratio strategies after perturbation of the stable age distribution. Journal of Theoretical Biology 186:213-221.

West, S. A., and B. C. Sheldon. 2002. Constraints in the evolution of sex ratio adjustment. Science 295:1685-1688.

West, S. A., D. M. Shuker, and B. C. Sheldon. 2005. Sex-ratio adjustment when relatives interact: a test of constraints on adaptation. Evolution 59:1211-1228.

Williams, G. C. 1979. The question of adaptive sex ratio in outcrossed vertebrates. Proceedings of the Royal Society B: Biological Sciences 205:567-580.
Associate Editor: Fredric J. Janzen Editor: Michael C. Whitlock 